\title{
CRICKETS, ZIPPERS, AND THE BERS UNIVERSAL TEICHMÜLLER SPACE
}

\author{
K. ASTALA AND F. W. GEHRING \\ (Communicated by Clifford J. Earle, Jr.) \\ Dedicated to Lipman Bers on his seventy-fifth birthday
}

\begin{abstract}
This paper contains a simple characterization of the conformal mappings $f$ of the unit disk $U$ whose Schwarzians lie in the closure of the Bers universal Teichmüller space. A second characterization is given and three examples are studied for the case where $f(U)$ is the complement of a quasiarc.
\end{abstract}

\section{INTRODUCTION}

Let $E$ denote the Banach space of functions $\phi$ analytic in the unit disk $U$ with norm

$$
\|\phi\|_{U}=\sup _{z \in U}|\phi(z)|\left(1-|z|^{2}\right)^{2},
$$

and let $S_{f}$ denote the Schwarzian derivative $\left(f^{\prime \prime} / f^{\prime}\right)^{\prime}-\frac{1}{2}\left(f^{\prime \prime} / f^{\prime}\right)^{2}$ of a locally conformal mapping $f$ of a domain in the extended complex plane $\overline{\mathbb{C}}$.

In the 1960s, Bers showed how all Teichmüller spaces $T(G)$ of Fuchsian groups $G$ or Riemann surfaces $U / G$ could be embedded in a very natural manner into a universal Teichmüller space $T$, the subset of $E$ consisting of Schwarzian derivatives $S_{f}$ of conformal mappings $f$ of $U$ which have a quasiconformal extension to $\widetilde{\mathbb{C}}$. Besides providing new insight into the spaces $T(G)$, this embedding raised the interesting question of how $T$ is related to the subset $S$ of $E$ of Schwarzians $S_{f}$ of all conformal mappings $f$ of $U$. For example, Bers asked if $S=\bar{T}$ and, later, if $S$ is itself connected. It turns out that $S$ is closed in $E$, that int $S$ is equal to $T$ [G2] and hence is connected [EE], that $S \backslash \bar{T}$ is not empty [G3], and that $S$ contains isolated points and thus is not connected [T, As].

Received by the editors June 5, 1989.

1980 Mathematics Subject Classification (1985 Revision). Primary 30C60.

This research was supported by grants from the National Science Foundation and the Academy of Finland. Both authors gratefully acknowledge support from the Mathematical Sciences Research Institute in Berkeley during the period 1 January to 1 July 1986 when the main results of this paper were obtained. 
In $\S 2$ of this paper we give a simple characterization of the conformal mappings of $U$ whose Schwarzians lie in $\bar{T}$; our approach is a modification of a method used in [AG]. We apply this result in $\S 3$ to obtain a second characterization for the case where $f(U)=\overline{\mathbb{C}} \backslash \alpha$ and $\alpha$ is a quasiarc. We consider three examples of this situation in $\S 4$ and show, in particular, that $S_{f}$ is not in $\bar{T}$ for a class of quasiarcs $\alpha$ which includes Thurston's simple zippers [T, §3]. We conclude in $\S 5$ with a remark on the relation between $T$ and the corresponding space $T_{1}$, where $S_{f}$ is replaced by $f^{\prime \prime} / f^{\prime}$.

\section{Closure of $T$}

Throughout this paper, all quasiconformal mappings are assumed to be sense preserving. Given a simply connected domain $D$ of hyperbolic type in $\overline{\mathbb{C}}$, we let $\rho_{D}$ denote the density of the hyperbolic metric with curvature -4 in $D$. Next, if $f$ is conformal in $D$, we set

$$
\left\|S_{f}\right\|_{D}=\sup _{z \in D}\left|S_{f}(z)\right| \rho_{D}(z)^{-2} \text {. }
$$

This is the counterpart of (1.1) for the domain $D$.

2.2. Theorem. Suppose that $f$ is a conformal mapping of $U$ onto $D$. Then $S_{f}$ is in $\bar{T}$ if and only iffor each $K>1$ there exists a homeomorphism $g$ of $D$ onto a quasidisk such that for each disk $B$ in $D, g \mid B$ has a $K$-quasiconformal extension to $\overline{\mathbb{C}}$.

Proof. Suppose that $S_{f}$ is in $\bar{T}$ and that $B$ is a disk in $D$. For each $K>1$, we can choose a conformal mapping $\phi$ of $U$ onto a quasidisk with

$$
\left\|S_{g}\right\|_{B} \leq\left\|S_{g}\right\|_{D}=\left\|S_{f}-S_{\phi}\right\|_{U} \leq 2 \frac{K-1}{K+1}
$$

where $g=\phi \circ f^{-1}$. Then by [L, Theorems II.4.1 and II.5.1], $g \mid B$ has a quasiconformal extension to $\overline{\mathbb{C}}$ with complex dilatation $\mu_{g}$ where

$$
\left\|\mu_{g}\right\|_{L^{\infty}} \leq \frac{1}{2}\left\|S_{f}\right\|_{D} \leq \frac{K-1}{K+1},
$$

hence establishing the necessity.

For the sufficiency, fix $0<\varepsilon<1$ and $c \geq 1$, choose $K>1$ so that

$$
\varepsilon=96 c^{2} \frac{K^{2}-1}{K^{2}+1},
$$

and let $g$ be the homeomorphism corresponding to $K$ in the hypotheses. Then $g$ is $K$-quasiconformal in a neighborhood of each point of $D \backslash\{\infty\}$ and hence in $D$; by the measurable Riemann mapping theorem, there exists an $h, K$ quasiconformal in $\overline{\mathbb{C}}$, such that $\psi=h \circ g$ is conformal in $D$.

Fix $z_{0}$ in $D \backslash\{\infty\}$ and let $B=B\left(z_{0}, r\right)$ be the disk with center $z_{0}$ and radius $r$ where $c r=\operatorname{dist}\left(z_{0}, \partial D\right)$. If $g_{B}$ is the extension of $g \mid B$, then 
$\psi_{B}=h \circ g_{B}$ is $K^{2}$-quasiconformal in $\overline{\mathbb{C}}, \psi_{B}$ agrees with $\psi$ in $B$, and

$$
\left|S_{\psi}\left(z_{0}\right)\right| \rho_{B}\left(z_{0}\right)^{-2} \leq\left\|S_{\psi}\right\|_{B} \leq 6 \frac{K^{2}-1}{K^{2}+1}=\frac{\varepsilon c^{-2}}{16}
$$

[L, Theorem II.3.2]. The Koebe distortion theorem implies that

$$
\rho_{D}\left(z_{0}\right)^{-1} \leq 4 \operatorname{dist}\left(z_{0}, \partial D\right) \leq 4 c \rho_{B}\left(z_{0}\right)^{-1}
$$

and hence that

$$
\left|S_{\psi}\left(z_{0}\right)\right| \rho_{D}\left(z_{0}\right)^{-2} \leq 16 c^{2}\left|S_{\psi}\left(z_{0}\right)\right| \rho_{B}\left(z_{0}\right)^{-2} \leq \varepsilon .
$$

Thus

$$
\left\|S_{f}-S_{\phi}\right\|_{U}=\left\|S_{\psi}\right\|_{D} \leq \varepsilon
$$

where $\phi=\psi \circ f, \phi(U)=\psi(D)$ is a quasidisk and $S_{f}$ is in $\bar{T}$.

2.3. Remark. The above proof shows that $S_{f}$ is in $\bar{T}$ if for some fixed $c \geq 1$ and each $K>1$ there exists a homeomorphism $g$ of $D$ onto a quasidisk such that $g \mid B$ has a $K$-quasiconformal extension to $\overline{\mathbb{C}}$ for all $B=B\left(z_{0}, r\right)$ in $D$ with $c r \leq \operatorname{dist}\left(z_{0}, \partial D\right)$.

For convenience of notation we let $S, T$, and $\bar{T}$ also denote the family of images $f(U)$ when $S_{f}$ is in $S, T$, and $\bar{T}$, respectively. With this convention, we see that if $g$ is a Möbius transformation, then $D$ is in $\bar{T}$ whenever $g(D)$ is in $\bar{T}$. We have the following useful extension of this observation:

2.4. Corollary. $A$ domain $D$ is in $\bar{T}$ if for each $K>1$ there exists a $K$ quasiconformal self-mapping $g$ of $\overline{\mathbb{C}}$ with $g(D)$ in $\bar{T}$.

Proof. By a distortion theorem for quasiconformal mappings, we can choose a constant $c \geq 1$ with the following property: If $B=B\left(z_{0}, r\right)$ is in $D \subset \mathbb{C}$ with $c r \leq \operatorname{dist}\left(z_{0}, \partial D\right)$ and if $g$ is a $K$-quasiconformal mapping of $D$ onto $D^{\prime} \subset \mathbb{C}$ with $1 \leq K \leq 2$, then $g(B)$ lies inside a disk $B^{\prime}$ in $D^{\prime}$. See [G1, p. 383] or [V1, Theorem 18.1].

Suppose $g$ is $K$-quasiconformal in $\overline{\mathbb{C}}$ with $1<K \leq 2$ and $D^{\prime}=g(D)$ in $\bar{T}$; as noted above, we may assume that $D$ and $D^{\prime}$ lie in $\mathbb{C}$. By Theorem 2.2 , there exists a homeomorphism $h$ of $D^{\prime}$ onto a quasidisk $D^{\prime \prime}$ such that $h \mid B^{\prime}$ has a $K$-quasiconformal extension to $\overline{\mathbb{C}}$ for each disk $B^{\prime}$ in $D^{\prime}$. Then $h \circ g(D)=D^{\prime \prime}$, and $h \circ g \mid B$ has a $K^{2}$-quasiconformal extension to $\overline{\mathbb{C}}$ for $B=B\left(z_{0}, r\right)$ in $D$ with $c r \leq \operatorname{dist}\left(z_{0}, \partial D\right)$.

\section{THE COMPLEMENT OF A QUASIARC}

Most of the known domains in $S \backslash \bar{T}$ are of the form $D=\overline{\mathbb{C}} \backslash \alpha$, where $\alpha$ is a quasiarc, i.e., the image of the closed positive half $I=[0, \infty]$ of the extended real axis $R$ under a quasiconformal mapping of $\overline{\mathbb{C}}$. (cf. [As, G3, T]. But see also [F].) We use Theorem 2.2 to derive the following criterion for the complement of a quasiarc to be in $\bar{T}$. 
3.1. Cricket Theorem. Suppose that $\alpha$ is a subarc of a quasicircle $\gamma$ in $\overline{\mathbb{C}}$. Then $D=\overline{\mathbb{C}} \backslash \alpha$ is in $\bar{T}$ if and only if for each $K>1$ there exists a sense preserving $K$-quasiconformal self-mapping $h$ of $\overline{\mathbb{C}}$ such that $h(z)=z$ in $\gamma \backslash \alpha$ and $h(\alpha) \cup \alpha$ is a quasicircle.

3.2. Remark. If we think of $\gamma$ as a band of spring steel, Theorem 3.1 says that $D=\overline{\mathbb{C}} \backslash \alpha$ is in $\bar{T}$ if and only if the part corresponding to $\alpha$ can be snapped by small deformations into positions which differ markedly from the original, while the remainder of the band remains fixed. This similarity to a metal toy cricket is the reason for the Theorem's name.

Proof of the necessity in Theorem 3.1. Let $G_{1}$ and $G_{2}$ denote the components of $\overline{\mathbb{C}} \backslash \gamma$. Then there exists a constant $a=a(\gamma)>0$ such that each $f$ conformal in $G_{j}$ with $\left\|S_{f}\right\|_{G_{j}}<a$ has a quasiconformal extension $g_{j}$ to $\overline{\mathbb{C}}$ with

$$
\left\|\mu_{g_{j}}\right\|_{L^{\infty}} \leq \frac{1}{a}\left\|S_{f}\right\|_{G_{j}}
$$

[L, Theorem II.4.1].

Fix $K>1$, and choose $0<\varepsilon<1$ so that $(1+\varepsilon) /(1-\varepsilon) \leq \sqrt{K}$. Because $D$ is in $\bar{T}$, there exists a conformal mapping $f$ of $D$ onto a quasidisk $D^{\prime}$ with

$$
\left\|S_{f}\right\|_{G_{j}} \leq\left\|S_{f}\right\|_{D} \leq a \varepsilon .
$$

By (3.3), $f \mid G_{j}$ has a quasiconformal extension $g_{j}$ to $\overline{\mathbb{C}}$ with maximal dilatation $K\left(g_{j}\right) \leq \sqrt{K}$. Then $h=g_{2}^{-1} \circ g_{1}$ is $K$-quasiconformal in $\overline{\mathbb{C}}, h$ fixes each point of $\gamma \backslash \alpha=\beta$, and

$$
g_{1}(\alpha) \cup g_{2}(\alpha)=\partial D^{\prime}
$$

Hence $h(\alpha) \cup \alpha=g_{2}^{-1}\left(\partial D^{\prime}\right)$ is a quasicircle.

The proof for the sufficiency in Theorem 3.1 requires several preliminary results.

3.4. Lemma. For each $0<a<\infty$, there exists $0<b=b(a)<\infty$ with the following property: If $g$ is $K$-quasiconformal in $\overline{\mathbb{C}}$, with $1 \leq K \leq e^{a / b}$, and if $g$ fixes $z_{1}, z_{2}, \infty$, then

$$
|g(z)-z| \leq b\left|z_{1}-z_{2}\right| \log K
$$

for $z$ in $B\left(z_{1}, a\left|z_{1}-z_{2}\right|\right)$.

Proof. The argument is similar to that for Lemma 7 in [G3]. Let $\rho_{G}$ and $h_{G}$ denote the hyperbolic density and metric with curvature -4 in $G=\overline{\mathbb{C}} \backslash$ $\{0,1, \infty\}$ and choose $b$ so that

$$
1=2 b \inf \left\{\rho_{G}(z): z \in G \cap \bar{B}(0,2 a)\right\} .
$$

Then, by a theorem of Teichmüller [Ah, pp. 53-61],

$$
h_{G}(g(z), z) \leq \frac{1}{2} \log K
$$

for each $K$-quasiconformal mapping $g$ of $\overline{\mathbf{C}}$ which fixes $0,1, \infty$. 
Suppose that $g$ satisfies the above hypotheses with $z_{1}=0$ and $z_{2}=1$. Fix $z \in G \cap B(0, a)$, and let $\gamma$ denote the hyperbolic geodesic joining $z$ and $g(z)$ in $G$ and $\ell(\gamma)$ the euclidean length of $\gamma$. Then $\gamma \subset \bar{B}(0,2 a)$ since otherwise

$$
a<\ell(\gamma \cap \bar{B}(0,2 a)) \leq 2 b \int_{\gamma} \rho_{G} d s=2 b h_{G}(g(z), z) \leq b \log K,
$$

contradicting our choice of $K$. Thus

$$
|g(z)-z| \leq \ell(\gamma) \leq 2 b \int_{\gamma} \rho_{G} d s \leq b \log K
$$

and we obtain (3.5) for the case where $z_{1}=0$ and $z_{2}=1$. The general case follows from applying what was proved above to

$$
h(w)=\frac{g(z)-z_{1}}{z_{2}-z_{1}}, \quad z=w\left(z_{2}-z_{1}\right)+z_{1} .
$$

A continuous map $g$ of $E \subset \mathbb{C}$ into $\mathbb{C}$ is s-quasisymmetric [V2], $0<s<$ $\infty$, if, for each three points $z, z^{\prime}, z^{\prime \prime}$ in $E$,

$$
s \leq\left|\frac{z^{\prime}-z}{z^{\prime \prime}-z}\right| \leq \frac{1}{s} \text { implies that }\left|\frac{g\left(z^{\prime}\right)-g(z)}{g\left(z^{\prime \prime}\right)-g(z)}\right| \leq\left|\frac{z^{\prime}-z}{z^{\prime \prime}-z}\right|+s .
$$

3.6. Lemma. For each $s>0$ there exist $t>0$ and $K>1$ with the following property: If $g$ is $K$-quasiconformal and satisfies $|g(z)-z| \leq \operatorname{tr}$ in $B\left(z_{0}, 2 r\right)$, then $g$ is s-quasisymmetric in $\bar{B}\left(z_{0}, r\right)$.

Proof. Since the composition of an $s$-quasisymmetric map with a euclidean similarity is $s$-quasisymmetric, we may assume $z_{0}=0$ and $r=1$.

Suppose that the conclusion of Lemma 3.6 does not hold for some $s>$ 0 . Then for each integer $j$ there exists a homeomorphism $g_{j}$ which is $K_{j}$ quasiconformal and satisfies $\left|g_{j}(z)-z\right| \leq t_{j}$ in $B(0,2)$, where $K_{j} \rightarrow 1$ and $t_{j} \rightarrow 0$. In addition, there exist points $z_{j}, z_{j}^{\prime}, z_{j}^{\prime \prime}$ in $\bar{B}(0,1)$ such that

$$
s \leq \frac{\left|z_{j}^{\prime}-z_{j}\right|}{\left|z_{j}^{\prime \prime}-z_{j}\right|} \leq \frac{1}{s} \text { and } \frac{\left|w_{j}^{\prime}-w_{j}\right|}{\left|w_{j}^{\prime \prime}-w_{j}\right|}-\frac{\left|z_{j}^{\prime}-z_{j}\right|}{\left|z_{j}^{\prime \prime}-z_{j}\right|}>s,
$$

where $w_{j}=g_{j}\left(z_{j}\right), w_{j}^{\prime}=g_{j}\left(z_{j}^{\prime}\right), w_{j}^{\prime \prime}=g_{j}\left(z_{j}^{\prime \prime}\right)$. By passing to a subsequence we may assume that $z_{j}, w_{j} \rightarrow z_{0}, z_{j}^{\prime}, w_{j}^{\prime} \rightarrow z_{0}^{\prime}, z_{j}^{\prime \prime}, w_{j}^{\prime \prime} \rightarrow z_{0}^{\prime \prime}$. Condition (3.7) then implies that $z_{0}=z_{0}^{\prime}=z_{0}^{\prime \prime}$.

Let $h_{j}=\psi_{j} \circ g_{j} \circ \phi_{j}^{-j}$, where $\phi_{j}$ and $\psi_{j}$ are euclidean similarities with

$$
\phi_{j}\left(z_{j}\right)=0, \quad \phi_{j}\left(z_{j}^{\prime \prime}\right)=1, \quad \psi_{j}\left(w_{j}\right)=0, \quad \psi_{j}\left(w_{j}^{\prime \prime}\right)=1 .
$$

Then $h_{j}$ is $K_{j}$-quasiconformal in

$$
B_{j}=\phi_{j}(B(0,2)) \supset B\left(0, r_{j}\right), \quad r_{j}=\left|z_{j}^{\prime \prime}-z_{j}\right|^{-1} .
$$


Since $h_{j}$ fixes 0,1 and omits $\infty$ in $B_{j}$, by passing to a second subsequence we may assume that $\left|h_{j}(z)\right| \rightarrow|z|$ locally uniformly in $\mathbb{C}$. Then $\left|\phi_{j}\left(z_{j}^{\prime}\right)\right|$ is bounded in $j$ by the first part of (3.7) and

$$
\frac{\left|w_{j}^{\prime}-w_{j}\right|}{\left|w_{j}^{\prime \prime}-w_{j}\right|}-\frac{\left|z_{j}^{\prime}-z_{j}\right|}{\left|z_{j}^{\prime \prime}-z_{j}\right|}=\left|h_{j}\left(\phi_{j}\left(z_{j}^{\prime}\right)\right)\right|-\left|\phi_{j}\left(z_{j}^{\prime}\right)\right| \rightarrow 0,
$$

which contradicts the second part of (3.7).

3.8. Lemma. For each $K>1$, there exists $s>0$ with the following property: If $g$ is an s-quasisymmetric homeomorphism of a closed disk $B$, then $g \mid B$ has a homeomorphic extension to $\overline{\mathbb{C}}$ which is $K$-quasiconformal in $\overline{\mathbb{C}} \backslash B$.

Proof. This follows from [TV, Theorems 5.23 and 2.6].

Proof of sufficiency in Theorem 3.1. By performing a preliminary Möbius transformation, we may assume that $\alpha$ has 0 and $\infty$ as its endpoints. Next, by hypothesis, there exists for each $K>1$ a $K$-quasiconformal self-mapping $h$ of $\overline{\mathbb{C}}$ such that $h(z)=z$ for $z$ in $\beta=\gamma \backslash \alpha$, and $h(\alpha) \cup \alpha$ is a quasicircle. Hence $h$ fixes 0 and $\infty$,

$$
h(\alpha) \cap \gamma=h(\alpha) \cap \alpha=\{0, \infty\}
$$

and we can label the components $G_{1}$ and $G_{2}$ of $\overline{\mathbb{C}} \backslash \gamma$ so that $h(\alpha) \subset G_{2} \cup$ $\{0, \infty\}$. Then the fact that $h$ is sense preserving and the identity on $\gamma \backslash \alpha$ implies that $h\left(G_{2}\right) \subset G_{2}$. Hence

$$
g(z)= \begin{cases}z & \text { if } z \in G_{1} \cup \beta, \\ h(z) & \text { if } z \in G_{2}\end{cases}
$$

defines a homeomorphism of $D=G_{1} \cup \beta \cup G_{2}$ onto $D^{\prime}=G_{1} \cup \beta \cup h\left(G_{2}\right)$, and $\partial D^{\prime}=h(\alpha) \cup \alpha$ is a quasicircle.

To complete the proof, it suffices to show that for each $B=B\left(z_{0}, r\right)$ in $D$ with $3 r \leq \operatorname{dist}\left(z_{0}, \partial D\right), g \mid B$ has a $K_{0}$-quasiconformal extension $g_{0}$ to $\overline{\mathbb{C}}$ where $K_{0}=K_{0}(K) \rightarrow 1$ as $K \rightarrow 1$. If $B \subset G_{1}$, we may take $g_{0}(z)=z$ and $K_{0}=1$; if $B \subset G_{2}$, we may choose $g_{0}(z)=h(z)$ and $K_{0}=K$.

It remains to consider the case where there exists a point $z_{1} \in B \cap \beta$. Since $\beta$ is unbounded, we can choose $z_{2} \in \beta$ with $\left|z_{1}-z_{2}\right|=r$. Then $h$ is $K$ quasiconformal in $\overline{\mathbb{C}}$ and fixes $z_{1}, z_{2}, \infty$. Hence, by Lemma 3.4 applied to $h$ in $B\left(z_{1}, 3 r\right)$, there exists an absolute constant $b>0$ such that

$$
|g(z)-z| \leq|h(z)-z| \leq t r, \quad t=b \log K
$$

if $z \in B\left(z_{0}, 2 r\right)$ and $1 \leq K \leq e^{3 / b}$. Since $g$ is $K$-quasiconformal in $B\left(z_{0}, 2 r\right)$, Lemma 3.6 implies that $g$ is $s$-quasisymmetric in $\bar{B}\left(z_{0}, r\right)$, where $s=s(K) \rightarrow 0$ as $K \rightarrow 1$. Then by Lemma 3.8, $g \mid B$ has a $K_{0}$-quasiconformal extension to $\overline{\mathbb{C}}$ where $K_{0}=K_{0}(s) \rightarrow 1$ as $s \rightarrow 0$.

\section{EXAMPLES}

We apply the Cricket Theorem to give two classes of quasiarcs $\alpha$ for which $D=\overline{\mathbb{C}} \backslash \alpha$ is in $\bar{T}$ and a third for which $D=\overline{\mathbb{C}} \backslash \alpha$ is not in $\bar{T}$. 
A quasiarc $\alpha$ is asymptotically conformal if, for each $K>1, \alpha=g(I)$, where $g$ is a quasiconformal in $\overline{\mathbb{C}}$ and $K$-quasiconformal in a neighborhood $V$ of $I=[0, \infty]$. If $\alpha \subset \mathbb{C}$ is asymptotically conformal, then

$$
\frac{\left|z_{1}-z_{2}\right|+\left|z_{2}-z_{3}\right|}{\left|z_{1}-z_{3}\right|} \rightarrow 1 \quad \text { as }\left|z_{1}-z_{3}\right| \rightarrow 0,
$$

for each ordered triple of points $z_{1}, z_{2}, z_{3}$ in $\alpha$.

4.1. Theorem. If $\alpha$ is an asymptotically conformal quasiarc, then $D=\overline{\mathbb{C}} \backslash \alpha$ is in $\bar{T}$.

Proof. Fix $K>1$. Then there exists $g$ quasiconformal in $\overline{\mathbb{C}}$ with $g K$ quasiconformal in a neighborhood $V$ of $I$ and $g(I)=\alpha$. Choose $0<a<\pi$ and $0<b<a$ so that

$$
W=\left\{z=r e^{i \vartheta}: 0 \leq r \leq \infty,|\vartheta| \leq a\right\} \subset V, \quad \frac{a}{a-b}=K
$$

and set

$$
f\left(r e^{i \vartheta}\right)=r e^{i \phi(\vartheta)}, \quad f(\infty)=\infty
$$

where $\phi$ is piecewise linear and increasing in $[-\pi, \pi]$ with $\phi( \pm \pi)= \pm \pi$, $\phi( \pm a)= \pm a$ and $\phi(0)=b$. Then $f$ is a $K$-quasiconformal self-mapping of $\overline{\mathbb{C}}, f(W)=W$ and $f(z)=z$ in $\overline{\mathbb{C}} \backslash W$.

Let $h=g \circ f \circ g^{-1}$. Then $h$ is $K^{3}$-quasiconformal in $g(V)$ and each point of $\overline{\mathbb{C}} \backslash g(W)$ has a neighborhood in which $h(z)=z$. Thus $h$ is $K^{3}$ quasiconformal in $\overline{\mathbb{C}}, h(\alpha) \cup \alpha$ is a quasicircle and $D$ is in $\bar{T}$ by Theorem 3.1 with $\gamma=g(R)$ and $R$ the real axis.

An arc $\alpha$ is a graph if for each line $\lambda$ parallel to some fixed line, the set $\alpha \cap \lambda$ is either connected or empty.

4.3. Theorem. If $\alpha$ is a quasiarc which is a graph, then $D=\overline{\mathbb{C}} \backslash \alpha$ is in $\bar{T}$.

Proof. We consider here only the case where $\alpha$ is unbounded. Then, by means of a preliminary similarity mapping, we may assume that $\alpha \cap \lambda$ is connected or empty for each line $\lambda$ parallel to the $y$-axis, that $\alpha$ has endpoints 0 and $\infty$, and that $\alpha$ does not meet the left half-plane.

Suppose that there exists a constant $1 \leq m<\infty$ such that

$$
\left|z_{1}-z_{2}\right| \leq m\left|\operatorname{Re}\left(z_{1}\right)-\operatorname{Re}\left(z_{2}\right)\right|
$$

for each $z_{1}, z_{2}$ in $\alpha \cap \mathbb{C}$. Given $K>1$, choose $t$ so that $(1+t) /(1-t)=K$, and let

$$
h(z)= \begin{cases}z+i t \operatorname{Re}(z) & \text { if } 0 \leq \operatorname{Re}(z)<\infty, \\ z & \text { if }-\infty<\operatorname{Re}(z) \leq 0,\end{cases}
$$

and $h(\infty)=\infty$. Then $h$ is $K$-quasiconformal in $\overline{\mathbb{C}}$ and (4.4) implies that $h(\alpha) \cup \alpha$ is a quasicircle. Hence $D=\overline{\mathbb{C}} \backslash \alpha$ is in $\bar{T}$ by Theorem 3.1.

For the general case, fix $K>1$ and let $\beta=(-\infty, 0)$ denote the negative half of the real axis. Then $\gamma=\alpha \cup \beta$ is a quasicircle. Let $f$ map the upper half-plane 
$H$ conformally onto the upper component $G$ of $\overline{\mathbb{C}} \backslash \gamma$ so that its homeomorphic extension to $\bar{H}$ fixes $\infty$. Because $\alpha$ is a graph, $\{z+i t: z \in G\} \subset G$ for $t>0$. Hence, if we let $\phi$ map $H$ conformally onto $U$ so that 0 and $\infty$ correspond to -1 and 1 , respectively, we can apply [HS, pp. 313-314] to $\psi=f \circ \phi^{-1}$ to conclude that $\operatorname{Re}\left(f^{\prime}(z)\right) \geq 0$ in $H$.

Now set

$$
g(z)=\int_{i}^{z} f^{\prime}(\zeta)^{t} d \zeta, \quad h=g \circ f^{-1},
$$

for $0<t<1$. Then

$$
\left\|S_{h}\right\|_{G}=\left\|S_{f}-S_{g}\right\|_{H} \leq(1-t) \sup _{z \in H}\left(\left|S_{f}(z)\right|+\frac{t}{2}\left|\frac{f^{\prime \prime}(z)}{f^{\prime}(z)}\right|^{2}\right) \rho_{H}(z)^{-2} \leq 24(1-t),
$$

$h$ has a $K$-quasiconformal extension to $\overline{\mathbb{C}}$ for $t$ near 1 , and

$$
\left|g^{\prime}(z)\right| \leq m \operatorname{Re}\left(g^{\prime}(z)\right), \quad m=\sec (\pi t / 2),
$$

in $H$. If $-\infty<\xi_{1}<\xi_{2}<\infty$ and $0<\eta<\infty$, then

$$
\begin{aligned}
\left|z_{1}-z_{2}\right| \leq \int_{\xi_{1}}^{\xi_{2}}\left|g^{\prime}(\xi+i \eta)\right| d \xi & \leq m \int_{\xi_{1}}^{\xi_{2}} \operatorname{Re}\left(g^{\prime}(\xi+i \eta)\right) d \xi \\
& =m\left|\operatorname{Re}\left(z_{1}\right)-\operatorname{Re}\left(z_{2}\right)\right|,
\end{aligned}
$$

where $z_{j}=g\left(\xi_{j}+i \eta\right)$, and letting $\eta \rightarrow 0$ yields $(4.4)$ for $z_{1}, z_{2}$ in $h(\alpha) \cap \mathbb{C}$. Thus $h(\overline{\mathbb{C}} \backslash \alpha)$ is in $\bar{T}$ and hence so is $\overline{\mathbb{C}} \backslash \alpha$ by Corollary 2.4.

Theorem 4.3 allows us to characterize those quasiarcs, which are invariant with respect to a homothety $f(z)=a z$, with complements in $\bar{T}$.

4.5. Corollary. Suppose that $\alpha$ is a quasiarc, that 0 is an interior point of $\alpha$, and that $f(\alpha) \subset \alpha$ for some $a$ in $B(0,1) \backslash\{0\}$. Then $D=\overline{\mathbb{C}} \backslash \alpha$ is in $\bar{T}$ if and only if $\alpha$ is a graph.

Proof. The necessity follows from [As, Theorem 4.1] and the sufficiency from Theorem 4.3.

The consideration of quasiarcs that are invariant with respect to a translation $f(z)=z+a$ leads us to the notion of an interlocking sequence. Given any triple of points $z_{j-1}, z_{j}, z_{j+1}$ in $\mathbb{C}$ we let

$$
r\left(z_{j}\right)=\frac{z_{j+1}-z_{j}}{z_{j-1}-z_{j}}
$$

Suppose next that $\alpha$ is a subarc of a quasicircle $\gamma$ which has 0 and $\infty$ as its endpoints. A sequence of distinct points $z_{j}$ in $\alpha$ is interlocking if there exist constants $0<a, b<\infty$, disjoint neighborhoods $V_{j}$ of $z_{j}$, and a component $G$ of $\overline{\mathbb{C}} \backslash \gamma$ with the following properties:

$$
\left|r\left(z_{j}\right)\right| \leq a \text { for } j \geq 2 .
$$




$$
\left\{\begin{array}{l}
\text { If } w_{j-1} \in G \cap V_{j-1}, w_{j} \in G \cap V_{j}, w_{j+1} \in G, \text { and }\left|r\left(z_{j}\right)-r\left(w_{j}\right)\right| \leq b \\
\text { for some } j \geq 2, \text { then } w_{j+1} \in V_{j+1} .
\end{array}\right.
$$

$$
\operatorname{dia}\left(V_{j}\right) /\left|z_{j}\right| \rightarrow 0 \quad \text { as } j \rightarrow \infty .
$$

Here $\operatorname{dia}\left(V_{j}\right)$ denotes the euclidean diameter of $V_{j}$.

4.9. Theorem. If $\alpha$ is a quasiarc that contains an interlocking sequence, then $D=\overline{\mathbb{C}} \backslash \alpha$ is not in $\bar{T}$.

Proof. By hypothesis there exist points $z_{j}$ in $\alpha$, neighborhoods $V_{j}$ of $z_{j}$, and a component $G$ of $\overline{\mathbb{C}} \backslash \gamma$ which satisfy (4.6) through (4.8); here $\gamma$ is any quasicircle which contains $\alpha$.

Suppose that $D=\overline{\mathbb{C}} \backslash \alpha$ is in $\bar{T}$. Then for each $K>1$ there exists a $K$ quasiconformal self-mapping $h$ of $\overline{\mathbb{C}}$ with $h(z)=z$ in $\gamma \backslash \alpha$ and $h(\alpha) \cup \alpha$ a quasicircle. By replacing $h$ by $h^{-1}$ if necessary, we may assume that

$$
w_{j}=h\left(z_{j}\right) \in h(\alpha \backslash\{0, \infty\}) \subset G, \quad j \geq 1 .
$$

Next, by Lemma 3.4, we can choose $K>1$ so that $w_{1} \in V_{1}$ and $w_{2} \in V_{2}$ and so that each $K$-quasiconformal self-mapping $g$ of $\overline{\mathbb{C}}$ which fixes $0,1, \infty$ satisfies

$$
|g(w)-w| \leq b,
$$

for $w$ in $B(0, a)$. Then (4.6) and (4.10) applied to

$$
g(w)=\frac{h(z)-w_{j}}{w_{j-1}-w_{j}}, \quad z=w\left(z_{j-1}-z_{j}\right)+z_{j},
$$

yield

$$
\left|r\left(w_{j}\right)-r\left(z_{j}\right)\right|=\left|g\left(r\left(z_{j}\right)\right)-r\left(z_{j}\right)\right| \leq b, \quad j \geq 2 .
$$

Since $w_{1} \in V_{1}$ and $w_{2} \in V_{2},(4.7)$ implies that $w_{j} \in V_{j}$ for all $j$ and hence that

$$
\lim _{j \rightarrow \infty} \frac{\left|h\left(z_{j}\right)-z_{j}\right|}{\left|z_{j}\right|}=0 .
$$

Because $h\left(z_{j}\right)$ and $z_{j}$ separate 0 and $\infty$ in $h(\alpha) \cup \alpha$, (4.11) then implies that $h(\alpha) \cup \alpha$ is not a quasicircle.

4.12. Example. For $\pi / 3<\vartheta<2 \pi / 3$ and $j \geq 1$, let $\alpha_{j}$ denote the polygonal arc formed by joining successively the points $2 j-2,2 j-1,2 j-1+e^{i(\pi-\vartheta)}$, $2 j+e^{i \vartheta}, 2 j$ with linear segments. Next let

$$
\alpha=\left(\bigcup_{j=1}^{\infty} \alpha_{j}\right) \cup\{\infty\},
$$

and let $\beta=(-\infty, 0)$ be the negative half of the real axis. Then $\gamma=\alpha \cup \beta$ is a quasicircle. Moreover, $\alpha$ contains an interlocking sequence when $\vartheta<\pi / 2, \alpha$ is a graph when $\vartheta \geq \pi / 2$, and hence $D=\overline{\mathbb{C}} \backslash \alpha$ is in $\bar{T}$ if and only if $\vartheta \geq \pi / 2$. 
Proof. Suppose that $\vartheta<\pi / 2$. We shall show that the points $z_{j}=j$ are interlocking in $\alpha$ with

$$
a=1, \quad b=\frac{\cos ^{2} \vartheta}{8}, \quad c=\frac{2 b}{\cos \vartheta}, \quad V_{j}=B(j, c)
$$

and $G$ the upper component of $\overline{\mathbb{C}} \backslash \gamma$. Since (4.6) and (4.8) hold trivially, we need only prove that, if $w_{j-1}, w_{j}, w_{j+1}$ satisfy the hypotheses in (4.7), then $d_{j+1}<c$ where $d_{k}=\left|w_{k}-k\right|$.

If $j$ is even, then

$$
\begin{aligned}
d_{j+1}-2 d_{j}-d_{j-1} & \leq\left|w_{j+1}-2 w_{j}+w_{j-1}\right| \\
& =\left|r\left(w_{j}\right)-r\left(z_{j}\right)\right|\left|w_{j}-w_{j-1}\right| \\
& \leq b\left|w_{j}-w_{j-1}\right| \leq b\left(d_{j}+1+d_{j-1}\right),
\end{aligned}
$$

and hence $d_{j+1} \leq \cos \vartheta$. From this it follows that

$$
\left|w_{j+1}-w_{j}\right|^{2} \leq\left|w_{j+1}-j\right|^{2} \leq 1-d_{j+1} \cos \vartheta
$$

while as above

$$
\begin{aligned}
(1-b)^{2} & \leq(1-b)^{2}\left|w_{j}-w_{j-1}\right|^{2} \\
& \leq\left(\left|w_{j}-w_{j-1}\right|-\left|w_{j+1}-2 w_{j}+w_{j-1}\right|\right)^{2} \\
& \leq\left|w_{j+1}-w_{j}\right|^{2} \leq 1-d_{j+1} \cos \vartheta
\end{aligned}
$$

Thus, $d_{j+1}<c$. If $j$ is odd, then

$$
\begin{aligned}
d_{j+1} \cos \vartheta+1+d_{j} \cos \vartheta & \leq\left|w_{j+1}-w_{j}\right| \\
& \leq\left|w_{j+1}-2 w_{j}+w_{j-1}\right|+\left|w_{j}-w_{j-1}\right| \\
& \leq(b+1)\left|w_{j}-w_{j-1}\right| \leq b+1,
\end{aligned}
$$

and $d_{j+1}<c$.

4.13. Remark. When $\vartheta<\pi / 2$, the arc $\alpha$ in Example 4.12 is a simple zipper in the sense of Thurston [T, $\S 3]$. Theorem 4.9 shows that the complements of all such quasiarcs are contained in $S \backslash \bar{T}$.

\section{A COUNTEREXAMPLE}

In [AG] we considered an alternative model for the Bers space $T$; namely, the set $T_{1}$ of logarithmic derivatives $f^{\prime \prime} / f^{\prime}$ of conformal mappings $f$ of $U$ into $\mathbb{C}$ with quasiconformal extensions to $\overline{\mathbb{C}}$ equipped with the norm

$$
\|\phi\|_{U}^{*}=\sup _{z \in U}|\phi(z)|\left(1-|z|^{2}\right) \text {. }
$$

Then $\pi(\phi)=\phi^{\prime}-\frac{1}{2} \phi^{2}$ is a continuous map of $T_{1}$ into $T, \pi\left(\bar{T}_{1}\right) \subset \bar{T}$, and it is natural to ask if $\bar{T}=\pi\left(\bar{T}_{1}\right)$. We give an example here to show that this is not the case, thus completing the proof of Theorem 3.4 in [AG]. There we exhibited 
a conformal mapping $f$ with $f^{\prime \prime} / f^{\prime}$ in $\pi^{-1}(\bar{T}) \backslash \bar{T}_{1}$ instead of $S_{f}=\pi\left(f^{\prime \prime} / f^{\prime}\right)$ in $\bar{T} \backslash \pi\left(\bar{T}_{1}\right)$, as asserted in Corollary 3.19. We are grateful to Dr. Y. Gatok for calling our attention to this oversight.

5.2. Theorem. There exists a conformal mapping $f$ of $U$ into $\mathbb{C}$ for which $S_{f}$ is in $\bar{T} \backslash \pi\left(\bar{T}_{1}\right)$.

Our proof is based on the following preliminary calculation:

5.3. Lemma. Suppose that $G=B \cup B^{\prime}$ where $B$ is an open disk, $B^{\prime}$ is an open disk or half-plane, and $\partial B$ and $\partial B^{\prime}$ meet in acute exterior angles. If $h$ is a homeomorphism of $\bar{G}$ which maps $G$ conformally into $\mathbb{C}$ and if

$$
\left|\frac{h^{\prime \prime}}{h^{\prime}}(z)\right| \leq c \operatorname{dist}(z, \partial G)^{-1}
$$

for $z$ in $G$, where $0<c<1$, then

$$
\frac{\operatorname{dia}(h(C))}{|h(a)-h(b)|} \geq \frac{1-c}{16}\left(\frac{\operatorname{dia}(C)}{|a-b|}\right)^{1-c}-1,
$$

where $C=\partial B \backslash B^{\prime}$ and $\{a, b\}=\partial B \cap \partial B^{\prime}$.

Proof. By performing a preliminary similarity mapping, we may assume that $B=B(i, r)$ and that $a=-s, b=s$ where $s>0$ and $s^{2}+1=r^{2}$. Then for $x$ in $I=(-s, s)$, $\operatorname{dist}(x, \partial G)=s-|x|$ and

$$
\log \left|\frac{h^{\prime}(x)}{h^{\prime}(0)}\right| \leq\left|\int_{0}^{x} \frac{h^{\prime \prime}}{h^{\prime}}(\xi) d \xi\right| \leq c \int_{0}^{|x|}(s-\xi)^{-1} d \xi=\log \left(1-\frac{|x|}{s}\right)^{-c}
$$

by (5.4). Hence

$$
\operatorname{dia}(h(I)) \leq \int_{-s}^{s}\left|h^{\prime}(x)\right| d x \leq \frac{2}{1-c}\left|h^{\prime}(0)\right| s .
$$

Similarly

$$
\log \left|\frac{h^{\prime}(0)}{h^{\prime}(i)}\right| \leq\left|\int_{0}^{1} \frac{h^{\prime \prime}}{h^{\prime}}(i y) d y\right| \leq c \int_{0}^{1}\left(y^{2}+s^{2}\right)^{-1 / 2} d y \leq \log 2\left(\frac{r}{s}\right)^{c}
$$

by (5.4) and we obtain

$$
\left|h^{\prime}(0)\right| \leq 2\left(\frac{r}{s}\right)^{c}\left|h^{\prime}(i)\right| \leq 8\left(\frac{r}{s}\right)^{c} \frac{\operatorname{dist}(h(i), h(C))}{\operatorname{dist}(i, \partial G)}
$$

from the Koebe distortion theorem. Since

$$
\operatorname{dia}(h(C)) \geq \operatorname{dist}(h(i), h(C))-\operatorname{dia}(h(I)),
$$

(5.5) follows from (5.6) and (5.7).

Proof for Theorem 5.2. Let $\left\{B_{j}\right\}$ be a sequence of open disks with centers in $H$ and diameter 1 such that $B_{j} \cap \partial H$ is an open interval $\left(a_{j}, b_{j}\right)$ and such that $\left|a_{j}-a_{k}\right| \geq 3$ for $j \neq k$ and

$$
\lim _{j \rightarrow \infty} \frac{\operatorname{dia}\left(C_{j}\right)}{\left|a_{j}-b_{j}\right|}=\infty, \quad C_{j}=\partial B_{j} \backslash L,
$$


where $L$ is the lower half-plane. Suppose next that $f$ maps $U$ conformally onto

$$
D=\left(\bigcup_{j=1}^{\infty} B_{j}\right) \cup L
$$

We shall show that $S_{f}$ is in $\bar{T} \backslash \pi\left(\bar{T}_{1}\right)$.

To show that $S_{f}$ is in $\bar{T}$, fix $K>1$, and for each $j$, let $g_{j}$ denote a Möbius self-mapping of $H$ for which $g_{j}\left(a_{j}\right)=0$ and $g_{j}\left(b_{j}\right)=\infty$. Next set

$$
h\left(r e^{i \vartheta}\right)= \begin{cases}r e^{i \vartheta / K} & \text { if } 0 \leq \vartheta<\pi, \\ r e^{i \vartheta} & \text { if }-\pi<\vartheta \leq 0,\end{cases}
$$

and

$$
g(z)= \begin{cases}g_{j}^{-1} \circ h \circ g_{j}(z) & \text { if } z \in B_{j}, \\ z & \text { if } z \in L .\end{cases}
$$

Then $g$ is a homeomorphism of $D$ onto a quasidisk. We must show $g \mid B$ has a $K_{0}$-quasiconformal extension $g_{0}$ to $\overline{\mathbb{C}}$ for each open disk $B$ in $D$ where $K_{0}(K) \rightarrow 1$ as $K \rightarrow 1$. If $B \subset L$, we may take $g_{0}(z)=z$. If $B \cap B_{j} \cap H \neq \varnothing$, then $g_{j}(B)$ lies in some half-plane

$$
G=\left\{r e^{i \vartheta}:|\vartheta-\phi|<\frac{\pi}{2}, 0<r<\infty\right\}, \quad|\phi|<\frac{\pi}{2},
$$

$h \mid G$ can be extended by means of a simple folding to a $K$-quasiconformal mapping $h_{0}$ of $\overline{\mathbb{C}}$, and $g_{0}=g_{j}^{-1} \circ h_{0} \circ g_{j}$ is the desired extension of $g \mid B$.

Suppose next that $S_{f}$ is in $\pi\left(\bar{T}_{1}\right)$. Then $S_{f}=\pi\left(\phi^{\prime \prime} / \phi^{\prime}\right)$ where $\phi^{\prime \prime} / \phi^{\prime}$ is in $\bar{T}_{1}$ and there exists a conformal mapping $\psi$ of $U$ onto a quasidisk $D^{\prime \prime} \subset \mathbb{C}$ with

$$
\sup _{z \in D^{\prime}}\left|\frac{h^{\prime \prime}}{h^{\prime}}(z)\right| \rho_{D^{\prime}}(z)^{-1}=\left\|\frac{h^{\prime \prime}}{h^{\prime}}\right\|_{D^{\prime}}^{*}=\left\|\frac{\phi^{\prime \prime}}{\phi^{\prime}}-\frac{\psi^{\prime \prime}}{\psi^{\prime}}\right\|_{U}^{*} \leq c<1,
$$

where $D^{\prime}=\phi(U)$ and $h=\psi \circ \phi^{-1}$. Hence

$$
\left|\frac{h^{\prime \prime}}{h^{\prime}}(z)\right| \leq c \operatorname{dist}\left(z, \partial D^{\prime}\right)^{-1}
$$

for $z$ in $D^{\prime}$.. Since $S_{f}=S_{\phi}$, there exists a Möbius transformation $g$ such that $\phi=g \circ f$ and hence $D^{\prime}=g(D)$. Then, since $\phi$ has no poles in $U, g(D) \subset \mathbb{C}$, $g(L)$ is an open disk or half-plane and $g\left(B_{j}\right)$ is an open disk for $j \geq j_{0}$.

Now fix $j \geq j_{0}$. Then $\partial g\left(B_{j}\right)$ and $\partial g(L)$ form acute exterior angles and

$$
\frac{\operatorname{dia}\left(g\left(C_{j}\right)\right)}{\left|g\left(a_{j}\right)-g\left(b_{j}\right)\right|} \geq \frac{\operatorname{dia}\left(C_{j}\right)}{\left|a_{j}-b_{j}\right|},
$$

where $a_{j}$ and $b_{j}$ are the endpoints of $C_{j}$. Thus we obtain

$$
\frac{\operatorname{dia}\left(h \circ g\left(C_{j}\right)\right)}{\left|h \circ g\left(a_{j}\right)-h \circ g\left(b_{j}\right)\right|} \geq \frac{1-c}{16}\left(\frac{\operatorname{dia}\left(C_{j}\right)}{\left|a_{j}-b_{j}\right|}\right)^{1-c}-1
$$


from (5.9), (5.10), and Lemma 5.3, with $B=g\left(B_{j}\right)$ and $B^{\prime}=g(L)$. Then (5.8) and (5.11) imply that $\partial D^{\prime \prime}=h \circ g(\partial D)$ is not a quasicircle, and we have a contradiction.

\section{REFERENCES}

[Ah] L. V. Ahlfors, Lectures on quasiconformal mappings, Van Nostrand, 1966.

[As] K. Astala, Selfsimilar zippers, Holomorphic Functions and Moduli I, Math. Sci. Res. Inst. Publ. 11 (1988), 61-73.

[AG] K. Astala and F. W. Gehring, Injectivity, the BMO norm and the universal Teichmüller space, J. Analyse Math. 46 (1986), 16-57.

[EE] C. J. Earle and J. Eells, On the differential geometry of Teichmüller spaces, J. Analyse Math. 19 (1967), 35-52.

[F] B. B. Flinn, Jordan domains and the universal Teichmüller space, Trans. Amer. Math. Soc. 282 (1984), 603-610.

[G1] F. W. Gehring, Rings and quasiconformal mappings in space, Trans. Amer. Math. Soc. 103 (1962), 353-393.

[G2] Univalent functions and the Schwarzian derivative, Comment. Math. Helv. 52 (1977), 561-572.

[G3] _ Spirals and the universal Teichmüller space, Acta Math. 141 (1978), 99-113.

[HS] W. Hengartner and G. Schober, On schlicht mappings to domains convex in one direction, Comment. Math. Helv. 45 (1970), 303-314.

[L] O. Lehto, Univalent functions and Teichmüller spaces, Springer-Verlag, 1986.

[T] W. P. Thurston, Zippers and univalent functions (The Bieberbach Conjecture: Proceedings of the Symposium on the Occasion of the Proof), Math. Surveys Monogr., vol. 21, Amer. Math. Soc., Providence, RI, pp. 185-197.

[TV] P. Tukia and J. Väisälä, Extension of embeddings close to isometries or similarities, Ann. Acad. Sci. Fenn. 9 (1984), 153-175.

[V1] J. Väisälä, Lectures on n-dimensional quasiconformal mappings, Lecture Notes in Math., vol. 229, Springer-Verlag, 1971.

[V2] _ Quasi-symmetric embeddings in euclidean spaces, Trans. Amer. Math. Soc. 264 (1981), 191-204.

Department of Mathematics, University of Helsinki, Helsinki, Finland

Department of Mathematics, University of Michigan, ANn Arbor, Michigan 48109 